\title{
The Population Genetics of Fungi: Tools and Techniques
}

\author{
Bruce A. McDonald
}

Department of Plant Pathology and Microbiology, Texas A\&M University, College Station 77843-2132.

Accepted for publication 22 January 1997.

Over the last 10 years plant pathologists have begun to realize that more knowledge about the genetic structure of populations of plant pathogens is needed to implement effective control strategies (48). Research on the genetic structure of fungal populations has mushroomed, and review papers that summarize these studies are numerous $(7,27,33,34,38)$. Although the number of fungal studies has increased greatly, the most comprehensive work has focused on a small number of plant-pathogenic fungi. The majority of these fungi can be recognized easily by their fruiting bodies or disease symptoms on aboveground plant parts. It has proven more difficult to assess the genetic structure of fungal populations that exist mainly belowground, because the distribution of individuals cannot be visualized directly and appropriate sampling procedures are less obvious and more cumbersome. Nevertheless, substantial progress has been made in interpreting the population genetic structure of some soilborne fungi $(1,17)$.

The purpose of this paper is to provide an overview of the tools and techniques of fungal population genetics. I will try to emphasize approaches that may be applied to studies of soilborne fungi. Instead of providing detailed methods, I will cite recent references where appropriate. There are many opinions regarding which techniques and tools are best suited to studies of fungal populations. I will give a personal and biased viewpoint, which I believe will be most useful to those who are just entering the field.

\section{Why Population Genetics?}

Why should plant pathologists study the population genetics of plant-pathogenic fungi? Because pathogens evolve. Pathogen populations must constantly adapt to changes in their environment to survive. In agricultural ecosystems, environmental changes may include resistant varieties, applications of fungicides and fertilizers, irrigation, and crop rotation. Plant pathologists have been aware of the ability of pathogen populations to evolve since Stakman described the wheat rusts as "shifty enemies." It is clear that agricultural systems impose strong directional selection on pathogen populations. Control strategies must target a population instead of an individual if they are to be effective. Thus, plant pathologists should focus more effort on understanding the genetics of populations to understand how populations will evolve in response to different control strategies.

Many factors contribute to genetic change (i.e., evolution) within populations. These factors include mutation, mating systems, gene flow or migration, population size, and selection. A thorough

Corresponding author: B. A. McDonald; E-mail address: b-mcdonald@tamu.edu

Additional keywords: DNA fingerprint, evolution, genetic diversity, RAPD, RFLP

Publication no. P-1997-0210-010

(C) 1997 The American Phytopathological Society discussion of the roles played by these factors can be found in population genetics textbooks (e.g., 19). In plant pathology, our ultimate goal should be to determine which factors play major roles in pathogen evolution and how these evolutionary forces interact to determine the genetic composition and evolutionary potential of pathogen populations.

\section{The Genetic Structure of Populations}

"Genetic structure" refers to the amount and distribution of genetic variation within and among populations. The majority of fungal studies to date have focused on the genetic structure of populations $(8,9,17,23,31,32,41)$. Defining the genetic structure of populations is a logical first step in studies of fungal population genetics because the genetic structure of a population reflects its evolutionary history and its potential to evolve. For evolution to occur by natural selection, there must be variation in fitness among individuals. Fisher's fundamental theorem of natural selection states that the mean fitness of a population is always increasing and that the rate of increase is proportional to the amount of additive genetic variation in fitness in a population (14). In more general terms, Fisher's theorem says that the evolutionary potential of a population is proportional to the amount of genetic diversity in a population. However, Fisher's theorem applies only to genes that are known to have an effect on fitness. Selectively neutral, DNA-based genetic markers such as restriction fragment length polymorphisms (RFLPs) or random amplified polymorphic DNAs (RAPDs) will not necessarily reflect the amount of genetic variation present in genes that condition fitness.

After elucidating the genetic structure of populations, it is appropriate to conduct experiments to differentiate among the effects of gene flow, drift, mating systems, and selection. The goal of these experiments is to determine which factors have the greatest impact on the population genetic structure of fungal populations. This is perhaps the most challenging aspect of deciphering fungal population genetics. It is difficult to design a field experiment that can differentiate among the various evolutionary forces that affect the genetic structure of populations. Plant pathologists to date have conducted few experiments to differentiate among these factors $(6,10,20,22,35)$.

\section{Some Important Questions}

I finish this section by listing some example questions that should be considered by plant pathologists as they begin to conduct experiments in population genetics with a novel pathogen. The first three questions relate to genetic structure: (i) How much genetic diversity exists in a population? (ii) How is genetic diversity distributed within populations (and on what spatial scale)? (iii) How is genetic diversity distributed among populations (and on what spatial scale)? The next three questions relate to the role of specific evolutionary processes: (iv) How do sexual and asexual repro- 
duction affect population structure? (v) How do migration and genetic drift affect population structure? (vi) How does selection affect population structure? The final three questions relate specifically to plant pathology: (vii) What is the best definition of a "population" for this pathogen? (i.e., What geographical and species boundaries can be defined by gene flow and mating system?) (viii) How do different control strategies affect the genetic structure of populations? (ix) What is the evolutionary potential of this pathogen? (i.e. Is it likely to evolve rapidly or slowly in a changing environment?)

Other important questions are likely to come up as knowledge of a pathosystem develops, but I believe that these nine questions offer a good starting point for focusing experiments on the population genetics of plant pathogens.

\section{THE TOOLS AND TECHNIQUES OF POPULATION GENETICS}

In this section, I arbitrarily organize the tools and techniques of population genetics into three general categories: (i) types of genetic markers; (ii) sampling methodologies; and (iii) analytical methods. I will try to emphasize approaches that may be most suitable for studies of soilborne fungi.

\section{Genetic Markers}

The choice of genetic marker can have a substantial impact on the analysis and interpretation of data. For questions relating to the roles played by population size, mating systems, and gene flow, selectively neutral genetic markers are preferred. For questions relating to the effects of selection, selected markers should be used. An exception to these guidelines exists when working with fungi that reproduce mainly asexually, producing a population structure that is composed largely of clonal lineages. In these cases, a neutral marker such as a DNA fingerprint may be used to address questions about selection because of the complete correspondence between genotype (e.g., DNA fingerprint) and phenotype (selected marker, e.g., pathotype). However, a complete correspondence between genotype and phenotype may not always be assumed, because several studies have shown that different pathotypes can arise within the same clonal lineage $(13,16)$, usually as a result of selection. Although the advantages and disadvantages of different types of genetic markers have been discussed elsewhere $(24,33,42)$, I will present a brief overview of some of these markers and their potential uses.

For most questions in population genetics, it is best to use genetic markers that are selectively neutral, highly informative, reproducible, and relatively easy (=inexpensive) to assay. Isozymes, RFLPs, and RAPDs have been used extensively in eucaryotes. Although there are now several studies on eucaryotes that provide evidence that isozymes are not always neutral (44), isozymes remain a potent genetic marker in fungi that possess sufficient variation at allozyme loci. However, because the majority of fungal studies currently are conducted with DNA-based genetic markers, I will focus on these. If the organism in question can be grown in pure culture on artificial media, the choice of DNA marker is open. If the organism cannot be grown on artificial media, then polymerase chain reaction (PCR)-based methods are likely to be more appropriate due to limitations on the amount of tissue that can be isolated for DNA extraction.

Before beginning large-scale population surveys, it is worthwhile to conduct preliminary screens of different types of genetic markers to gauge their relative information content. Different categories of genetic markers may provide widely varying interpretations of the genetic structure of populations. For example, in Mycosphaerella graminicola, we found little diversity in its mitochondrial (mt) DNA worldwide, suggesting limited genotype diversity and a clonal population structure, whereas there was a large amount of diversity for nuclear RFLP loci, suggesting high levels of gene diversity and random mating populations (37). We also showed that DNA fingerprints based on hybridization of one probe to repetitive DNA sequences had greater resolution to differentiate clones then multilocus haplotypes based on hybridization of many single-copy probes for M. graminicola and Phaeosphaeria nodorum $(4,36)$. The greater resolution of DNA fingerprints in these cases likely reflects the larger number of loci assayed by the repetitive probes. My experience suggests that it is best to use the widest practical array of genetic markers to obtain the most comprehensive understanding of the genetic structure of populations. If practical, a mixture of selected and neutral markers encompassing both nuclear and $\mathrm{mt}$ genomes should be used.

The number of marker loci that should be assayed varies depending on the objectives of the investigator and the resources available. To obtain an accurate assessment of population genetic structure, I recommend using 6 to 12 unlinked marker loci distributed across many chromosomes. The rationale for using markers that are on different chromosomes is to assay a representative sample of variation in the nuclear genome and to permit analyses of gametic disequilibrium. Depending on allele frequencies at individual loci, up to 20 genetic markers may be needed to differentiate among clones with a high degree of confidence. For narrowly defined selection experiments (e.g., resistance and sensitivity to a fungicide), one marker may suffice.

\section{RAPDs}

RAPD markers (47) have become popular because this PCR technology is relatively easy to implement. Of greater importance to plant pathologists, RAPDs can be assayed using very small amounts of fungal biomass, making them an ideal tool for obligate biotrophs such as rusts and mildews. Because a large number of amplicons can be screened in a relatively short period of time, RAPDs are especially useful in differentiating clonal lineages for fungi that reproduce asexually. Furthermore, RAPD data are easy to interpret because they are based on amplification or nonamplification of specific DNA sequences (amplicons), producing a binary data set that is easy to enter into a spreadsheet for analysis.

Unfortunately, RAPDs have many drawbacks that must be weighed against their relative ease of use. There are several technical limitations associated with RAPDs that make them difficult to reproduce between labs and sometimes within labs. Some of these limitations can be overcome with proper controls, such as replicate DNA preparations, Southern analysis, and conversion of RAPD amplicons into sequence characterized amplified regions (SCARs [40]). There also are analytical problems associated with RAPDs (28). RAPDs have only two alleles (amplification or nonamplification) for each amplicon locus. Although this is ideal for genetic mapping, it is a drawback for measures of genetic diversity affected by the number of alleles at a locus. RAPDs are dominant, so they cannot differentiate homozygotes and heterozygotes without a progeny test. This is not an issue with haploid fungi, but it can be a problem with many basidiomycetes and oomycetes that are heterokaryons, diploid or polyploid. Some of the analytical problems resulting from dominance and two alleles per locus can be overcome by increasing the number of individuals assayed (by 2 to $10 \times$ compared to isozymes and RFLPs) and using only RAPD amplicon loci that have intermediate allele frequencies (28).

Despite their drawbacks, RAPDs are powerful tools that are especially useful for fungi that are obligate parasites or that have a population structure composed of clonal lineages. PCR-based genetic markers that can detect more than two alleles and that exhibit codominance, such as SCARs and microsatellites (18), are likely to replace RAPDs as studies of fungal population genetics become more sophisticated. As advanced PCR-based markers become available, it may become possible to amplify specific DNA sequences from soil or root samples and make a direct assessment of the genetic structure of populations of soilborne fungi without first making pure cultures. But if a fungus can be cultured readily 
on artificial media, then RFLPs offer many advantages for population genetics studies.

\section{RFLPs}

RFLPs in nuclear and $\mathrm{mt}$ genomes have been used in many studies of plant-pathogenic fungi. Because RFLPs are based on DNA-DNA hybridization, they are technically more difficult than RAPDs but offer the advantage of being more reproducible. Like RAPDs, RFLPs are easy to interpret. In addition, they are codominant and exhibit a potentially unlimited number of alleles per locus. For example, we found over 20 alleles on average for $10 \mathrm{nu}-$ clear RFLP loci among 2,000 isolates in an international collection of the wheat leaf blotch pathogen M. graminicola (R. E. Pettway and B. A. McDonald, unpublished data) and 11 alleles at these same loci among 700 isolates sampled from a single field (37).

One disadvantage of RFLPs is that they require relatively large amounts (5 to $10 \mu \mathrm{g}$ ) of DNA from each individual, so the fungus must be grown in pure culture prior to DNA extraction. Of greater concern for many plant pathologists, RFLP analysis requires more technical expertise, including cloning, Southern blotting, and labeling of probes. Once the compulsory techniques have been mastered, RFLP analysis can proceed very rapidly. As an example, we routinely collect data for two RFLP loci from 300 individuals in a week. Another disadvantage of RFLPs is that they require expensive nylon membranes for Southern blotting. If only a few loci are assayed, the cost of materials and supplies for RFLP analysis can be expensive compared to RAPD analysis. However, repeat hybridizations of the same blot brings costs down very quickly, in our lab to less than 30 cents per locus per individual (B. A. McDonald, unpublished data).

RFLPs are most useful for fungi that can be grown easily in pure culture. Because probes can be chosen to show a high degree of specificity to the target fungus, some contamination by DNA from plants, other fungi, or bacteria can be tolerated. The properties of codominance and multiple alleles make RFLP markers advantageous compared to RAPDs for most studies in population genetics. An added advantage is that other labs can reproduce results using the same battery of probes and restriction enzymes, allowing work to be shared and compared among several labs.

\section{Other Genetic Markers}

The term DNA fingerprint too often is used improperly to refer to any complex pattern of DNA bands on a gel. Before a banding pattern can be classified correctly as a DNA fingerprint, a thorough statistical analysis is needed to validate its unique properties. The analysis generally is simple, based on using frequencies of individual bands within populations to calculate the probability that two randomly chosen individuals will share the same band pattern. DNA fingerprints based on both RFLPs and RAPDs have been widely used in fungi (42). Their primary use is to identify clones or clonal lineages with a high degree of confidence. Their utility in population genetics can be extended beyond identification of clones by conducting genetic analysis to determine the inheritance of individual restriction fragments or amplicons in the DNA fingerprint. DNA fingerprints undoubtedly will be as useful for soilborne fungi as they have been for other fungi.

Amplified fragment length polymorphisms (AFLPs) offer a potentially powerful tool to detect polymorphic DNA sequences in fungi. AFLPs share many characteristics with RAPDs. They are dominant and usually only have two alleles per locus. AFLPs have an advantage over RAPDs because more loci are screened in each reaction and the longer primers make it more likely that an AFLP will be reproducible. AFLPs are likely to be useful for DNA fingerprinting applications because a large number of loci can be screened in one reaction. The disadvantages of AFLPs are that they require more technical expertise than RAPDs (ligations, restriction enzyme digestions, and polyacrylamide gels), and they suffer the same analytical limitations as RAPDs.
The mt genome can offer an independent perspective on the population genetics of a fungus. The mt genome is inherited independently of the nuclear genome, exhibits little or no recombination, and usually is inherited from only one of the parents. As a result of the lack of recombination and uniparental inheritance, mt genomes exist as series of clonal lineages that can be used to define lines of descent or paths of gene flow. Because the mt genome is small and exists in multiple copies, it offers a good target for PCR. RFLPs in mtDNA are assayed easily after purifying the mtDNA through cesium chloride ultracentrifugation. The purified mtDNA can be used as a probe that hybridizes to the entire mtDNA genome. We found low levels of variation in the mtDNA of the oak wilt pathogen Ceratocystis fagacearum that corresponded with the low level of variation in the nuclear genome, reinforcing our interpretation that this pathogen represents a recent introduction to North America or a recent speciation event (25).

In addition to mtDNA, it appears that many fungi possess extrachromosomal, cytoplasmically transmitted nucleic acids, such as double-stranded RNAs (dsRNAs) or mt plasmids. We have found dsRNAs at low frequencies $(0.5$ to $2 \%)$ in populations of six plant-pathogenic fungi studied in my lab. Although it might be tempting to use dsRNAs as genetic markers to define clonal lineages or to assess the frequency of anastomosis in natural populations, I believe they have too many drawbacks to be useful as genetic markers in population studies. If they occur at a low frequency (e.g., <5\%), a large number of individuals must be assayed to make meaningful comparisons among populations. Perhaps more importantly, dsRNAs are not likely to be stable genetic markers. They can be "cured" from clonal lineages by high temperatures or through formation of sexual spores. It is likely to require a great expenditure of resources (cloning and sequencing) to validate dsRNAs or mt plasmids as genetic markers for population studies, and they are not likely to offer any advantages over mtDNA.

Vegetative and mycelial compatibility groups (VCGs or MCGs) have been very useful for identifying clones of fungi that are largely asexual (26), although it has been shown that isolates with the same VCG are not always members of the same clone (3). The primary advantage of VCG analysis is that it is relatively easy and inexpensive to implement. A significant disadvantage is that VCGs are phenotypes instead of genotypes and, thus, are not useful for measuring population similarity and differentiation. Another disadvantage is that some isolates will not form nitrogen nonutilizing (nit) mutants.

Electrophoretic karyotypes based on pulsed field gel electrophoresis have been useful for determining the linkage relationships among RFLP and RAPD markers, especially in fungi that do not go through the sexual cycle in laboratories. Many plant-pathogenic fungi exhibit a high degree of variability for karyotypes in natural populations $(21,39)$, but the biological significance of this variability is not clear. The primary limitations of electrophoretic karyotypes are that they require a significant degree of technical expertise and the data are relatively expensive to produce. Boehm et al. (5) used electrophoretic karyotypes in conjunction with VCG analysis of 118 isolates of Fusarium oxysporum $\mathrm{f}$. sp. cubense to differentiate clonal lineages within this banana pathogen.

\section{Sampling Methods}

Most of the analyses conducted by population geneticists are based on frequencies of alleles (markers) at discrete genetic loci. Because analyses are based on allele frequencies, it is vital that sample sizes be adequate to make reliable estimates. Estimates of allele frequencies based on sample sizes of 3 to 10 individuals per population are usually meaningless, whereas estimates based on sample sizes of 30 to 100 individuals can be quite reliable. In general, the sampling strategy and sample size are adjusted according to the question being addressed by the experiment. Sample sizes must be large enough to allow meaningful statistical tests to be performed. Questions relating to genetic distance between popula- 
tions may be addressed adequately with a sample size as small as 30 to 40 individuals per population. Questions relating to selection coefficients may require hundreds or thousands of individuals per experimental unit, depending on the intensity of selection in the experiment and the number of generations assayed. Reference texts such as Weir's (45) can provide rough guidelines for determining the appropriate sample size.

Although the genetic tools described previously and the analytical tools summarized later are likely to be very similar for any pathogen, the sampling methodology is likely to be quite different for soilborne fungi than it is for fungi that exist mainly aboveground. Unless a soilborne fungus produces aboveground fruiting bodies (e.g., mushrooms), it is impossible to visualize directly the distribution of the fungi in the soil. An exception exists for fungi that can be visualized by symptomatic hosts when a uniform crop is grown, such as for Rhizoctonia bare patch of cereals (29). Soil is not a homogeneous environment, and it is unlikely that soilborne fungi will be distributed uniformly throughout a soil profile. The distribution of fungal individuals will be affected by soil depth and type, arrangement of host root systems, and levels of soil moisture and organic matter. In agricultural ecosystems, soil mixing is likely to disrupt the natural distributions that would be found in an undisturbed habitat.

Although it may be impossible to predict the distribution of fungi in soil beforehand, knowledge of the spatial distribution of soil types and host plants and of the life cycle of the fungus may assist in designing an appropriate sampling strategy. For example, if a fungus reproduces via zoospores, it would be useful to know whether a field floods regularly to provide a mechanism for longdistance (tens of meters) dispersal of the fungus. Zoospore dispersal may be affected by soil type, with greater movement in sandy rather than clay soils. A sampling strategy for a fungus that produces propagules distributed solely in soil probably is not suitable for a fungus that produces airborne spores with the potential to move long distances. The ultimate goal is to design a sampling strategy that will allow the researcher to determine the extent of movement of individual clones within populations and, eventually, to determine the geographic boundaries of distinct populations. In the case of ancient Armillaria clones, the appropriate scale of sampling was in the order of tens of meters (43). In the case of soil fungi with very limited dispersal capabilities, the appropriate scale of sampling may be on the order of centimeters.

There is no single best sampling scheme that will give a representative, random sample for all fungal populations. In the absence of any prior knowledge of population structure, it is best to utilize a hierarchical sampling scheme $(22,37)$ as a starting point to define the spatial scale that covers the dispersal distance of the fungus. An example of a 2-D hierarchical sampling scheme is shown in Figure 1; in samples taken from soils, investigators may wish to add soil depth as a third dimension. The hierarchy can be adjusted to larger or smaller spatial scales after interpreting the results from preliminary studies. For example, if the preliminary study shows that a single genotype is present at 1-m intervals across a 20 - $\mathrm{m}$ transect, the investigator would want to increase the sampling scale to $10-\mathrm{m}$ intervals along a $200-\mathrm{m}$ transect or move several kilometers and sample another field. Depending on the interest of the investigator, the hierarchy can include different soil strata or types, agricultural and nonagricultural soils (17), or different plant parts (22), locations in a field (32), or regions on a continent (23). After preliminary studies have shown the distribution of genotypes at several levels in the hierarchy, it will be easier to make additional collections that better define the boundaries of populations.

Ultimately, the sampling methodology will place limits on the interpretation of data. If collections are made only from diseased plants, then the biological interpretation can extend only to that fraction of the fungal population that caused disease on the particular cultivar from which the collection was made. If samples are drawn at random from soil, the interpretation can be extended only to that soil horizon from which the sample was drawn. It eventually becomes important to add a temporal dimension to a sampling scheme to assess the time scale over which genetic change occurs. A single sample drawn at one point in time represents a snapshot of a population. Many samples drawn across several generations are needed to develop a motion picture that reflects the dynamics of the evolutionary process.

\section{Analytical Methods}

The basic analytical methods of population genetics have been widely published in the genetics literature, with most of the standard analyses described in textbooks (e.g., 19,45). Due to the historical development of population genetics, the analytical tools were developed initially for diploid organisms. Fortunately, most of these analytical methods can be adapted easily to haploid systems. Rather then attempting to publish a compendium of analytical methods and formulas in this overview, I will use examples from fungal literature that in turn cite the original sources. A good resource for formulas used in genetic data analysis is Weir's book Genetic Data Analysis II (45). Many of the basic computations of population genetics can be performed on a desktop computer by a spreadsheet program. Although it is relatively easy to calculate basic population genetic parameters for a population, it can be more difficult to develop a biological interpretation of the statistics. It is always useful to discuss results with a population geneticist.

The first analysis performed on a population quantifies genetic diversity. For organisms like fungi that undergo both asexual and sexual reproduction, it is necessary to differentiate between diversity at individual loci, "gene diversity," and diversity based on the number of genetically distinct individuals in a population, "genotype diversity." Taken together, gene and genotype diversity constitutes genetic diversity. Measures of gene and genotype diversity are among the easiest and most informative parameters to quantify. Measures of gene diversity are based on the number and

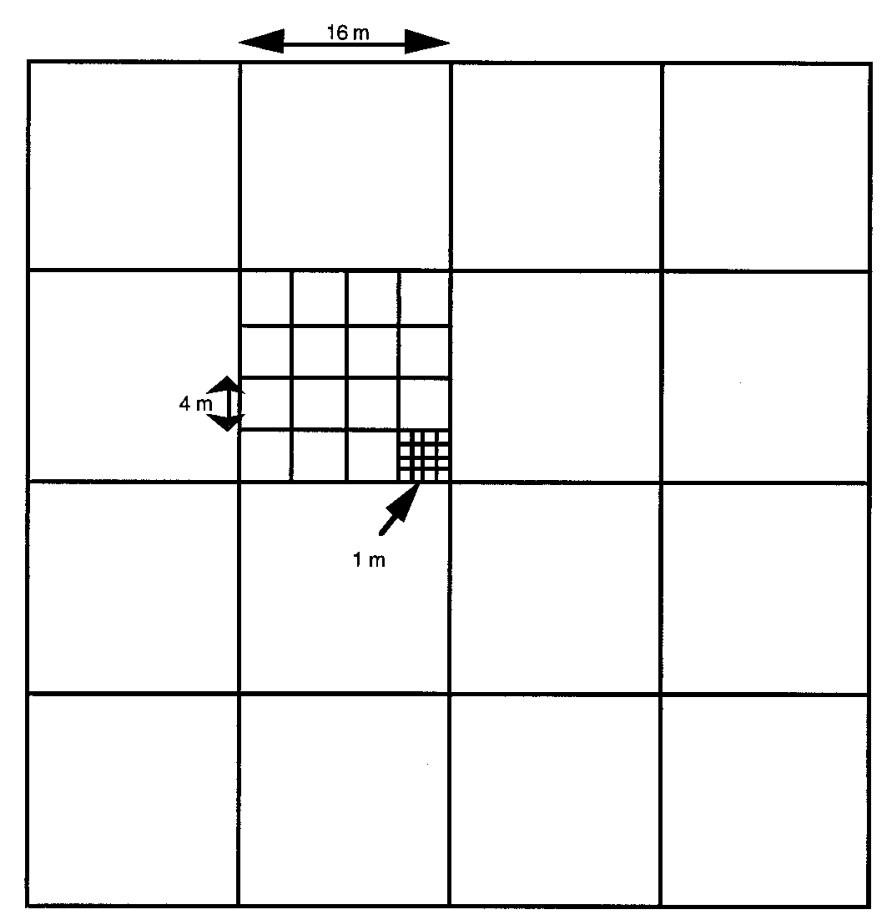

Fig. 1. Example of a hierarchical sampling design that could be used for preliminary determination of the genetic structure of a field population of a soilborne fungus. Samples are drawn at the intersections of each set of lines. In this case, collections would be made in a grid pattern at three spatial scales: 16,4 , and $1 \mathrm{~m}$. In total, 67 collections would be made within a field. For finer scale resolution, multiple isolations could be made from each soil core, bait, or plant part. 
frequencies of alleles at a locus (46). Gene diversity is affected by the age of a population, population size, and selection. For neutral loci, populations that have evolved over a long time at one location are expected to have more alleles than populations that have moved into an area more recently, because there has been more time for mutation to introduce new variants and for genetic drift to increase the frequencies of new alleles to detectable levels. Thus, a "center of origin" from which a species originates would be expected to have a higher level of gene diversity than other populations because it is older. In contrast, populations with low gene diversity may have been affected by large reductions in populations size (bottlenecks) or founder effects that eliminated many alleles. If the number of reproducing individuals remain small, then genetic drift occurs, and gene diversity will remain low. For genes in diploid organisms that exhibit overdominance (heterozygote advantage), selection may maintain several alleles in a population at intermediate frequencies.

Fungi exhibit a wide array of reproductive strategies that have an impact on their population biology (2). An important factor for many plant-pathogenic fungi is the relative abundance of sexual and asexual reproduction within populations. Asexual reproduction leads to clonal lineages that may exhibit a limited spectrum of virulence within each lineage (49). Sexual reproduction creates many new genotypes and allows new and existing virulence genes to be recombined into many different genetic backgrounds with each sexual generation. As a result, resistance gene pyramids are less likely to be effective against plant-pathogenic fungi that undergo sexual reproduction in nature. Measures of genotype diversity can offer insight into the relative abundance of sexual and asexual reproduction. Genotype diversity is based on the number of genotypes and the frequencies of genotypes in a population $(10,12)$. Genotype diversity is meaningful only for fungi that undergo some degree of asexual reproduction. DNA fingerprints (9) or multilocus genotypes (8) are needed to differentiate genotypes in a population. Measures of genotype diversity can provide an approximation of the fraction of a population that results from asexual reproduction, hence it is a potential indicator of the relative abundance of sexual and asexual reproduction. Selection also influences genotype diversity because genotypes that have the highest fitness increase in frequency over time. Knowledge of the spatial distribution of genotypes within populations can provide information about dispersal potential within fields $(10,22)$. Because soilborne fungi are likely to show a less homogeneous distribution than aerially disseminated fungi, spatial autocorrelation and other geostatistics are likely to be especially important (22).

If measures of genotype diversity indicate that sexual reproduction may be occurring, the hypothesis of random mating can be tested by measures of gametic disequilibrium (11). These tests require unlinked genetic markers, relatively large sample sizes (hundreds to thousands of isolates), and an appropriate sampling method. If the scale of sampling is too small, it is more likely that clones will be over-represented, and it may be useful to "clonecorrect" the data set $(11,37)$.

Gene diversity is not affected by the mating system. Fungi that reproduce exclusively through asexual reproduction may have as many alleles at individual loci as those that undergo a regular sexual cycle. The difference in mating system is reflected by measures of genotype diversity. For instance, assume a sample of 100 isolates is taken from a population that consists of a single clone that is heterozygous for the marker locus. In this case, gene diversity would be relatively high, with both alleles present at a frequency of 0.50 . However, genotype diversity would be very low, with only 1 genotype present among 100 isolates.

Comparisons among populations can be useful for defining population boundaries. In particular, plant pathologists would like to know whether quarantine measures are effective. If they are not, it would be useful to be able to determine the source of introduction and paths of migration among populations. In the ab- sence of movement of gametes or individuals among populations, the expectation is that genetic drift will lead to random changes in allele frequencies for neutral loci in different populations. If there is limited movement of genes among populations, we expect to find a correlation between genetic and geographic distance among populations. Gene flow can be measured either directly or indirectly (30). Direct measures are based on finding the same genotypes in different populations, as has been found for Phytophthora infestans (15) and Sclerotinia sclerotiorum (23). Indirect measures are based on comparison of allele frequencies in different populations and estimation of the degree of population subdivision (4). Both methods can be used to define the geographic boundaries of populations, as determined by absence of gene flow among populations. If no genotypes are shared between populations and the gene flow statistic is $N m<1$, this suggests that populations are not exchanging genes or genotypes and that quarantine measures have been effective.

\section{CONCLUSIONS}

The approaches used to elucidate the population genetics of soilborne fungi differ little from the approaches used to study aboveground fungi. The main difference is likely to be in sampling strategies and, ultimately, in the interpretation of the results. Hierarchical sampling should provide insight into the genetic structure of populations of fungi that exist belowground, just as plant pathologists recently have gained insight into the structure of populations that exist aboveground. The methods of population genetics offer powerful tools to elucidate the life histories of important plant pathogens and address fundamental questions about the biology of these organisms. Increased knowledge of the population biology of pathogens is likely to lead to better management of disease in agricultural ecosystems.

\section{ACKNOWLEDGMENTS}

This work was supported by the Texas Agricultural Experiment Station (Hatch Project 6928), the National Science Foundation (grant DEB9306377), and the USDA National Research Initiative Competitive Grants Program (grant 93-37303-9039).

\section{LITERATURE CITATIONS}

1. Anderson, J. B., and Kohn, L. M. 1995. Clonality in soilborne, plantpathogenic fungi. Annu. Rev. Phytopathol. 33:369-391.

2. Anderson, J. B., Kohn, L. M., and Leslie, J. F. 1992. Genetic mechanisms in fungal adaptation. Pages 73-98 in: The Fungal Community: Its Organization and Role in the Ecosystem. G. C. Carroll and D. T. Wicklow, eds. Marcel Dekker, Inc., New York.

3. Appel, D. J., and Gordon, T. R. 1996. Relationships among pathogenic and nonpathogenic isolates of Fusarium oxysporum based on the partial sequence of the intergenic spacer region of the ribosomal DNA. Mol. Plant-Microbe Interact. 9:125-138.

4. Boeger, J. M., Chen, R. S., and McDonald, B. A. 1993. Gene flow between geographic populations of Mycosphaerella graminicola (anamorph Septoria tritici) detected with RFLP markers. Phytopathology 83:11481154.

5. Boehm, E. W. A., Ploetz, R. C., and Kistler, H. C. 1994. Statistical analysis of electrophoretic karyotype variation among vegetative compatibility groups of Fusarium oxysporum f. sp. cubense. Mol. Plant-Microbe Interact. 7:196-207.

6. Brown, J. K. M., O’dell, M., Simpson, C. G., and Wolfe, M. S. 1990. The use of DNA polymorphisms to test hypotheses about a population of Erysiphe graminis f. sp. hordei. Plant Pathol. 39:391-401.

7. Burdon, J. J. 1993. The structure of pathogen populations in natural plant communities. Annu. Rev. Phytopathol. 31:305-323.

8. Burdon, J. J., and Jarosz, A. M. 1992. Temporal variation in the racial structure of flax rust (Melampsora lini) populations growing on natural stands of wild flax (Linum marginale): Local versus metapopulation dynamics. Plant Pathol. 41:165-179.

9. Chen, D., Zeigler, R. S., Leung, H., and Nelson, R. J. 1995. Population structure of Pyricularia grisea at two screening sites in the Philippines. Phytopathology 85:1011-1020. 
10. Chen, R. S., Boeger, J. M., and McDonald, B. A. 1994. Genetic stability in a population of a plant pathogenic fungus over time. Mol. Ecol. 3: 209-218.

11. Chen, R. S., and McDonald, B. A. 1996. Sexual reproduction plays a major role in the genetic structure of populations of the fungus Mycosphaerella graminicola. Genetics 142:1119-1126.

12. Drenth, A., Goodwin, S. B., Fry, W. E., and Davidse, L. C. 1993. Genotypic diversity of Phytophthora infestans in the Netherlands revealed by DNA polymorphisms. Phytopathology 83:1087-1092.

13. Drenth, A., Whisson, S. C., Maclean, D. J., Irwin, J. A. G., Obst, N. R., and Ryley, M. J. 1996. The evolution of races of Phytophthora sojae in Australia. Phytopathology 86:163-169.

14. Fisher, R. A. 1930. The Genetical Theory of Natural Selection. 1st ed. Clarendon Press, Oxford.

15. Goodwin, S. B., Cohen, B. A., and Fry, W. E. 1994. Panglobal distribution of a single clonal lineage of the Irish potato famine fungus. Proc. Natl. Acad. Sci. USA 91:11591-11595.

16. Goodwin, S. B., Sujkowski, L. S., and Fry, W. E. 1995. Rapid evolution of pathogenicity within clonal lineages of the potato late blight disease fungus. Phytopathology 85:669-676.

17. Gordon, T. R., Okamoto, D., and Milgroom, M. G. 1992. The structure and interrelationship of fungal populations in native and cultivated soils. Mol. Ecol. 1:241-249.

18. Groppe, K., Sanders, I., Wiemken, A., and Boller, T. 1995. A microsatellite marker for studying the ecology and diversity of fungal endophytes (Epichloe spp.) in grasses. Appl. Environ. Microbiol. 61:3943-3949.

19. Hartl, D. L., and Clark, A. G. 1989. Principles of Population Genetics. 2nd ed. Sinauer Associates, Sunderland, MA.

20. Jarosz, A. M., and Burdon, J. J. 1991. Host-pathogen interactions in natural populations of Linum marginale and Melampsora lini: II. Local and regional variation in patterns of resistance and racial structure. Evolution 45:1618-1627.

21. Kistler, H. C., and Miao, V. P. W. 1992. New modes of genetic change in filamentous fungi. Annu. Rev. Phytopathol. 30:131-152.

22. Kohli, Y., Brunner, L. J., Yoell, H., Milgroom, M. G., Anderson, J. B., Morrall, R. A. A., and Kohn, L. M. 1995. Clonal dispersal and spatial mixing in populations of the plant pathogenic fungus, Sclerotinia sclerotiorum. Mol. Ecol. 4:69-77.

23. Kohli, Y., Morrall, A. A., Anderson, J. B., and Kohn, L. M. 1992. Local and trans-Canadian clonal distribution of Sclerotinia sclerotiorum on canola. Phytopathology 82:875-880.

24. Kohn, L. M. 1992. Developing new characters for fungal systematics: An experimental approach for determining the rank of resolution. Mycologia 84:139-153.

25. Kurdyla, T. M., Guthrie, P. A. I., McDonald, B. A., and Appel, D. N. 1995. RFLPs in mitochondrial and nuclear DNA indicate low levels of genetic diversity in the oak wilt pathogen Ceratocystis fagacearum. Curr. Genet. 27:373-378.

26. Leslie, J. F. 1993. Fungal vegetative compatibility. Annu. Rev. Phytopathol. 31:127-151.

27. Leung, H., Nelson, R. J., and Leach, J. E. 1993. Population structure of plant pathogenic fungi and bacteria. Adv. Plant Pathol. 10:157-205.

28. Lynch, M., and Milligan, B. G. 1994. Analysis of population genetic structure with RAPD markers. Mol. Ecol. 3:91-99.

29. MacNish, G. C., and Neate, S. M. 1996. Rhizoctonia bare patch of cereals: An Australian perspective. Plant Dis. 80:965-971.

30. McDermott, J. M., and McDonald, B. A. 1993. Gene flow in plant pathosystems. Annu. Rev. Phytopathol. 31:353-373.
31. McDermott, J. M., McDonald, B. A., Allard, R. W., and Webster, R. K. 1989. Genetic variability for pathogenicity, isozyme, ribosomal DNA and colony color variants in populations of Rhynchosporium secalis. Genetics 122:561-565.

32. McDonald, B. A., and Martinez, J. P. 1990. DNA restriction fragment length polymorphisms among Mycosphaerella graminicola (anamorph Septoria tritici) isolates collected from a single wheat field. Phytopathology 80:1368-1373.

33. McDonald, B. A., and McDermott, J. M. 1993. The population genetics of plant pathogenic fungi. BioScience 43:311-319.

34. McDonald, B. A., McDermott, J. M., Goodwin, S. B., and Allard, R. W. 1989. The population biology of host-pathogen interactions. Annu. Rev. Phytopathol. 27:77-94.

35. McDonald, B. A., Mundt, C. C., and Chen, R. S. 1996. The role of selection on the genetic structure of pathogen populations: Evidence from field experiments with Mycosphaerella graminicola on wheat. Euphytica 92:73-86.

36. McDonald, B. A., Miles, J., Nelson, L. R., and Pettway, R. E. 1994. Genetic variability in nuclear DNA in field populations of Stagonospora nodorum. Phytopathology 84:250-255.

37. McDonald, B. A., Pettway, R. E., Chen, R. S., Boeger, J. M., and Martinez, J. P. 1995. The population genetics of Septoria tritici (teleomorph Mycosphaerella graminicola). Can. J. Bot. 73(Supp1.):S292-S301.

38. Milgroom, M. G. 1995. Analysis of population structure in fungal plant pathogens. Pages 213-230 in: Disease Analysis Through Genetics and Biotechnology: Interdisciplinary Bridges to Improved Sorghum and Millet Crops. J. F. Leslie and R. A. Frederiksen, eds. Iowa State University Press, Ames.

39. Mills, D., and McCluskey, K. 1990. Electrophoretic karyotypes of fungi: The new cytology. Mol. Plant-Microbe Interact. 3:351-357.

40. Paran, I., and Michelmore, R. W. 1993. Development of reliable PCRbased markers linked to downy mildew resistance genes in lettuce. Theor. Appl. Genet. 85:985-993.

41. Peever, T. L., and Milgroom, M. G. 1994. Genetic structure of Pyrenophora teres populations determined with random amplified polymorphic DNA markers. Can. J. Bot. 72:915-923.

42. Rosewich, U. L., and McDonald, B. A. 1994. DNA fingerprinting in fungi. Methods Mol. Cell. Biol. 5:41-48.

43. Smith, M. L., Bruhn, J. N., and Anderson, J. B. 1994. Relatedness and spatial distribution of Armillaria genets infecting red pine seedlings. Phytopathology 84:822-829.

44. Watt, W. B. 1994. Allozymes in evolutionary genetics: Self-imposed burden or extraordinary tool? Genetics 136:11-16.

45. Weir, B. S. 1995. Genetic Data Analysis II. Sinauer Associates, Sunderland, MA.

46. Welz, H. G., Kohler, W., and Leonard, K. J. 1994. Isozyme variation within and among pathogenic races of Cochliobolus carbonum on corn in North Carolina. Phytopathology 84:31-38.

47. Williams, J. G. K., Kubelik, A. R., Livak, K. J., Rafalski, J. A., and Tingey, S. V. 1990. DNA polymorphisms amplified by arbitrary primers are useful as genetic markers. Nucleic Acids Res. 18:6531-6535.

48. Wolfe, M. S., and Caten, C. E., eds. 1987. Populations of Plant Pathogens: Their Dynamics and Genetics. Blackwell Scientific Publications, Oxford.

49. Zeigler, R. S., Cuoc, L. X., Scott, R. P., Bernardo, M. A., Chen, D. H., Valent, B., and Nelson, R. J. 1995. The relationship between lineage and virulence in Pyricularia grisea in the Philippines. Phytopathology 85: 443-451. 